\title{
Serotonin and serotonin transporter in the rectum of patients with irritable bowel disease
}

\author{
MAGDY EL-SALHY $^{1,2}$, INGVILD WENDELBO ${ }^{1,2}$ and DORIS GUNDERSEN ${ }^{3}$ \\ ${ }^{1}$ Section for Gastroenterology, Department of Medicine, Stord Helse-Fonna Hospital, 5416 Stord; \\ ${ }^{2}$ Section for Gastroenterology, Institute of Medicine, University of Bergen, 5029 Bergen; \\ ${ }^{3}$ Department of Research, Helse-Fonna, 5528 Haugesund, Norway
}

Received May 14, 2013; Accepted May 31, 2013

DOI: $10.3892 / \mathrm{mmr} .2013 .1525$

\begin{abstract}
Irritable bowel syndrome (IBS) is a common chronic gastrointestinal disorder, which considerably reduces the quality of life of patients and represents an economic burden to society. In previous studies, the density of serotonin-expressing cells in the rectum of IBS patients did not differ from that of control subjects. The present study was undertaken to investigate the immunoreactivity intensity of serotonin and serotonin-selective reuptake transporter (SERT) in the rectum of IBS patients. A cohort of 50 patients with IBS (41 females and 9 males) were included in the study. Thirty patients had diarrhoea (IBS-D) and 20 had constipation (IBS-C) as the predominant symptom. Twenty-seven subjects were included as controls (19 females and 8 males). Rectal biopsy specimens were immunostained using the avidin-biotin complex method for serotonin and SERT. The immunoreactivity intensity was quantified by computerised image analysis using Olympus cell Sens imaging software. There was no statistical difference of serotonin immunoreactivity intensity in multiple comparisons between controls, IBS-total, IBS-D and IBS-C. Dunn's post test did not reveal any statistical differences among the four groups. There was a significant statistical difference in multiple comparisons between controls, IBS-total, IBS-D and IBS-C regarding the SERT immunoreactivity intensity. SERT immunoreactivity intensity of IBS-total, IBS-D and IBS-C differed significantly from that of controls. It was concluded that the reduced rectal SERT in the IBS patients could be one of the factors contributing to the development of both diarrhoea and constipation in these patients, and that the increasing body of evidence of a
\end{abstract}

Correspondence to: Professor Magdy El-Salhy, Section for Gastroenterology, Department of Medicine, Stord Helse-Fonna Hospital, Tysevegen 64, 5416 Stord, Norway

E-mail: magdy.el-salhy@helse-fonna.no

Key words: diarrhoea, constipation, irritable bowel syndrome, immunohistochemistry, serotonin, serotonin-selective reuptake transporter genetic abnormality involving SERT underlines the importance of the role of SERT in the pathophysiology of IBS.

\section{Introduction}

Irritable bowel syndrome (IBS) is a chronic gastrointestinal disorder with a prevalence of 5-20\% and an incidence of 200 per 100,000 of the adult population (1-9). It affects mostly individuals $<50$ years of age with a female predominance $(2,10-21)$. The diagnosis of IBS is based on assessment of symptoms as described in Rome III criteria. These criteria include recurrent abdominal pain/discomfort for at least 3 days per month during the previous 3 months associated with at least two of the following symptoms: improvement with defecation, onset associated with a change in the frequency of stools and/or a change in the form and appearance of stools $(22,23)$. In addition to these criteria, warning symptoms such as age $>50$ years, short history of symptoms, nocturnal symptoms, weight loss, rectal bleeding, anaemia, and the presence of markers for inflammation or infections should be excluded $(22,23)$. Based on the stool pattern, patients with IBS are subdivided into four subtypes, namely diarrhoea-predominant (IBS-D), constipation-predominant, mixed diarrhoea and constipation (M-IBS) and unclassified IBS (U-IBS) $(22,23)$.

IBS is not known to be associated with the development of serious disease or with increased mortality (24-27). However, it considerably reduces the quality of life in IBS patients and is an economic burden to society caused by overconsumption of healthcare resources such as diagnostic tests, medications and frequent hospitalisation as well as lower work productivity $(9,13,20,28-30)$.

Abnormal endocrine cells have been reported in the stomach, duodenum, ileum, colon and rectum of patients with IBS (unpublished data, 31-43). In a previous study (unpublished data), there was no difference in the rectal serotonin cell density between IBS patients (both IBS-D and IBS-C) and healthy subjects. It remains to be determined, however, whether the serotonin cell content in these patients and serotonin-selective reuptake transporter (SERT) are affected. The present study was therefore undertaken to investigate the intensity of immunoreactivity of serotonin and serotonin transporter in the same cohort of IBS patients studied earlier (unpublished data). 


\section{Materials and methods}

Patients and controls. A cohort of 50 patients with IBS that fulfilled Rome III criteria (22) were included in the study. The patients comprised 41 females and 9 males with a mean age of 37 years (range, 18-64 years). Thirty patients had IBS-D and twenty had IBS-C. All of the patients had experienced their symptoms for many years and they could not associate the onset of IBS symptoms with any events, in particular gastrointestinal infection. All of the patients were submitted to a complete physical examination and were investigated by means of blood (full blood count, electrolytes, calcium and inflammatory markers), liver, and thyroid function tests. The patients also underwent a gastroscopy with biopsy samples from the duodenum and celiac disease was excluded.

Twenty-seven additional subjects that underwent colonoscopy with rectal biopsies were used as controls. The colonoscopy was performed in 20 of these subjects because of gastrointestinal bleeding, where the source of bleeding was identified as haemorrhoids $(n=18)$ or angiodysplasia $(n=2)$ and in 7 due to health concerns caused by a relative(s) being diagnosed with colon carcinoma. These subjects were 19 females and 8 males with a mean age of 49 years (range, 18-68 years). All of these subjects were healthy and had no gastrointestinal complaints other than those stated above. The study was performed in accordance with the Declaration of Helsinki and was approved by the local Committee for Medical Research Ethics. All subjects gave both oral and written consents for participating in the study.

Colonoscopy, histopathology and immunohistochemistry. Colonoscopy was performed on both patients and controls, and biopsy samples were taken from the ileum and different segments of the colon. Four biopsy samples were also taken from the dorsal wall of the rectum $\sim 15 \mathrm{~cm}$ from the anus. These biopsy samples were fixed overnight in $4 \%$ buffered paraformaldehyde, embedded in paraffin, and cut into $5-\mu \mathrm{m}$ sections. The sections were stained with haematoxylin and eosin, and immunostained by the avidin-biotin complex $(\mathrm{ABC})$ method using the Vectastain $\mathrm{ABC}$ kit (Vector Laboratories, Inc., Burlingame, CA, USA). The sections were hydrated and immersed in $0.01 \%$ hydrogen peroxide in PBS buffer ( $\mathrm{pH}$ 7.4) for $10 \mathrm{~min}$ to inhibit endogenous peroxidase. After washing in buffer, the sections were treated with $1 \%$ bovine serum albumin for 30 min to block the non-specific binding sites, and then incubated with the primary antiserum/antibody at room temperature for $1 \mathrm{~h}$. The sections were then washed in PBS buffer and incubated with biotinylated swine anti-mouse IgG diluted 1:100 for $30 \mathrm{~min}$ at room temperature. After washing the slides in PBS buffer, the sections were incubated for $30 \mathrm{~min}$ with avidin-biotin-peroxidase complex diluted 1:100, and then immersed in 3,3'-diaminobenzidine (DAB) peroxidase substrate (Vector Laboratories, Inc.), followed by counterstaining in haematoxylin. The primary antibodies used were monoclonal mouse anti-serotonin (code no. 5HT-209; Dako, Carpinteria, CA, USA) and mouse anti-human serotonin transporter (Abcam, code no. ab1125; Abcam, Cambridge, MA, USA). The antibodies were used at dilutions of 1:1,500 and 1:500, respectively.

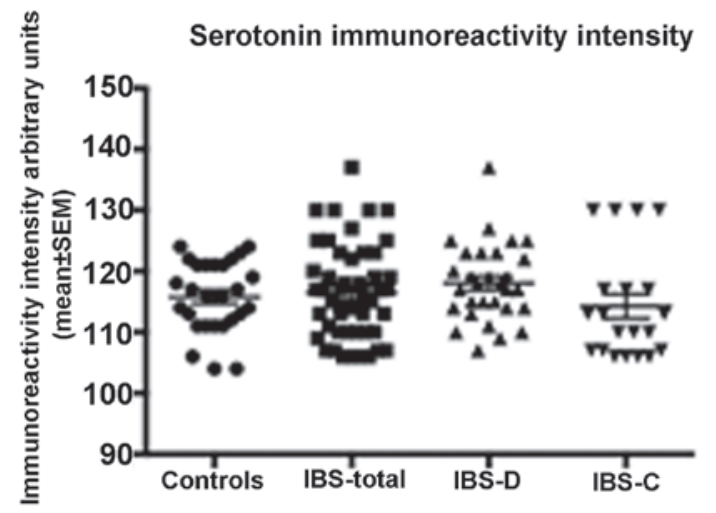

Figure 1. Serotonin immunoreactivity intensity in controls, irritable bowel syndrome (IBS)-total, IBS-D and IBS-C.

Computerised image analysis. The immunoreactivity (colour) intensities of serotonin and SERT were measured using Olympus cell Sens imaging software (version 1.7) on a computer linked to an Olympus microscope type BX43 with an Olympus camera (DP 26). A x20 objective was used, for which each frame (field) shown on the monitor represented an area of $0.14 \mathrm{~mm}^{2}$ of the tissue in each field. In each individual, serotonin and SERT were measured in five randomly chosen fields. Immunostained sections from IBS patients and controls were coded and mixed, and all measurements were made by the same person (M.E.) without knowledge of the identity of the sections.

Statistical analysis. Differences between the controls, all IBS patients (IBS-total), and IBS-D and IBS-C patients were tested by the Kruskal-Wallis non-parametric test with Dunn's test as post-test. The data were presented as mean \pm standard error values. Differences with $\mathrm{P}<0.05$ were considered to be statistically significant.

\section{Results}

Endoscopy and histopathology. The ileum, colon and rectum of both the patients and the control subjects were macroscopically normal. The results of the histopathological examinations of the ileum, colon and rectum were normal in both the patients and the controls.

Immunohistochemistry and computerized image analysis. In both patients and control subjects, serotonin-immunoreactive cells were identified mostly in the upper part of the crypts of Lieberkühn. These cells were basket- or flask-shaped. SERT immunoreactivity was observed in all the lining epithelium of rectum in both patients and control subjects.

Serotonin immunoreactive intensity. The immunoreactivity intensity of serotonin in controls was $115.8 \pm 1.1$. The corresponding numbers for IBS-total, IBS-D and IBS-C were $116.5 \pm 1.1,118.0 \pm 1.2$ and $114.3 \pm 2.0$, respectively. There was no statistical difference in the multiple comparisons between controls, IBS-total, IBS-D and IBS-C ( $\mathrm{P}=0.13)$. Dunn's post test did not reveal any statistical differences among the four groups ( $\mathrm{P}>0.05$ for all) (Figs. 1 and 2). 
A

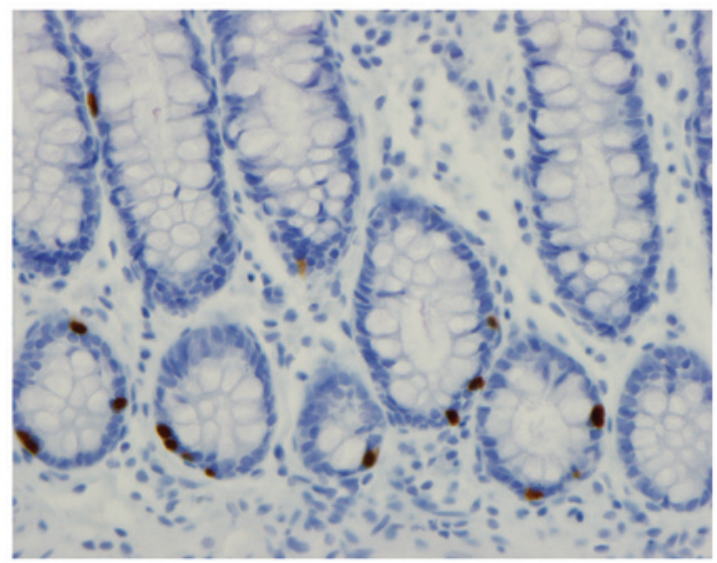

B

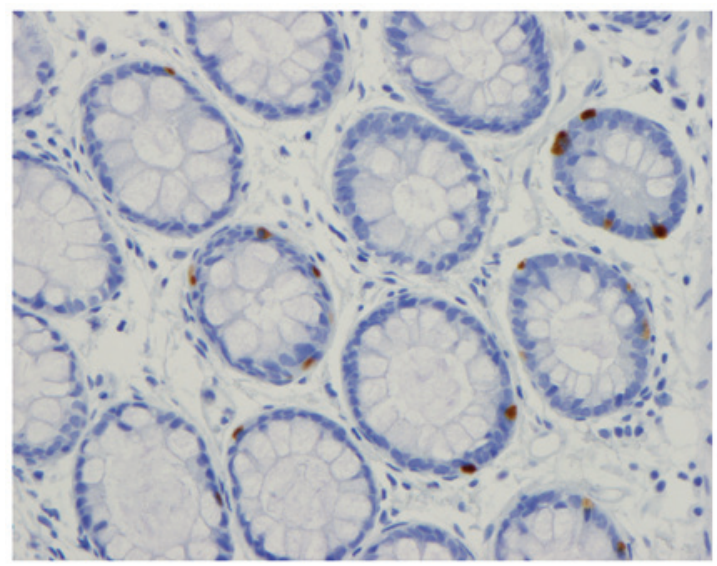

Figure 2. Serotonin immunoreactive cells in (A) the rectum of a control subject and (B) in a patient with irritable bowel syndrome.

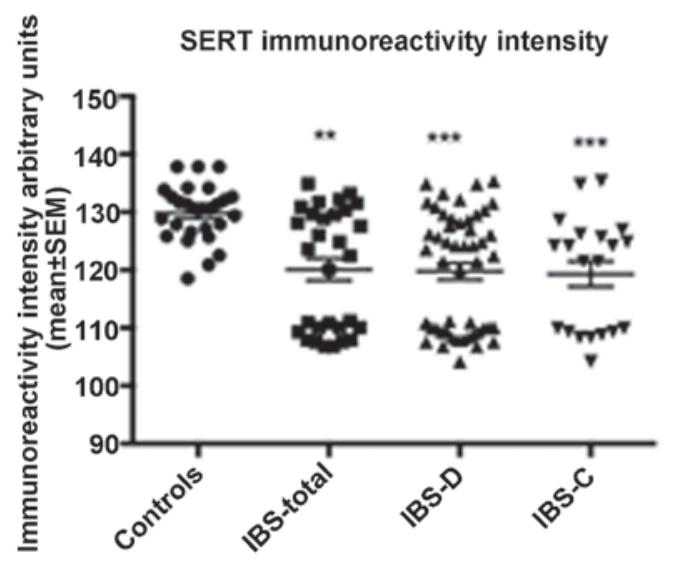

Figure 3. Serotonin-selective reuptake transporter (SERT) immunoreactivity intensity in rectal epithelial cells of control subjects, irritable bowel syndrome (IBS)-total, IBS-D and IBS-C. ${ }^{* *} \mathrm{P}<0.01$ and ${ }^{* * *} \mathrm{P}<0.0001$.

SERT immunoreactivity intensity. The immunoreactivity intensity of SERT in controls was $129.8 \pm 0.9$ and in IBS-total, IBS-D and IBS-C $120.1 \pm 1.9,119.6 \pm 1.4$ and $119.3 \pm 2.2$, respectively (Figs. 3 and 4). There was a significant statistical difference in the multiple comparisons between controls, IBS-total, IBS-D and IBS-C ( $\mathrm{P}<0.0001)$. A significant difference was observed between the SERT immunoreactivity intensity of IBS-total, IBS-D and IBS-C and the controls $(<0.01,<0.001$ and $<0.001$, respectively) (Figs. 3 and 4$)$.

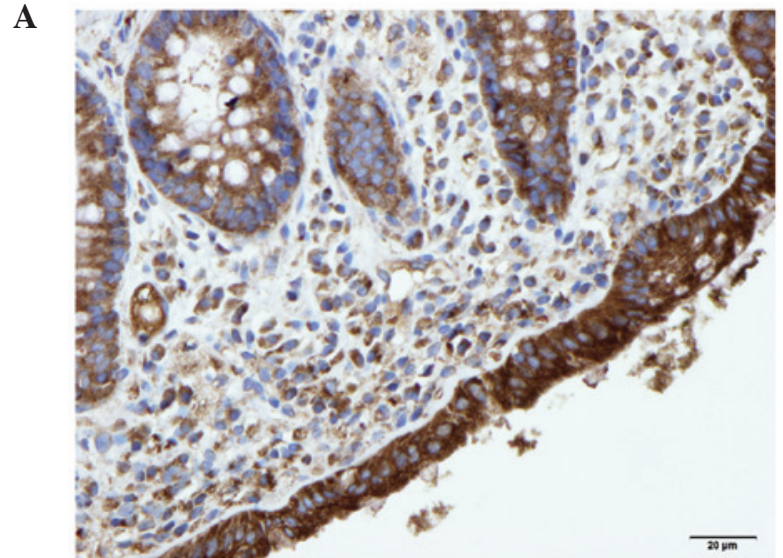

B

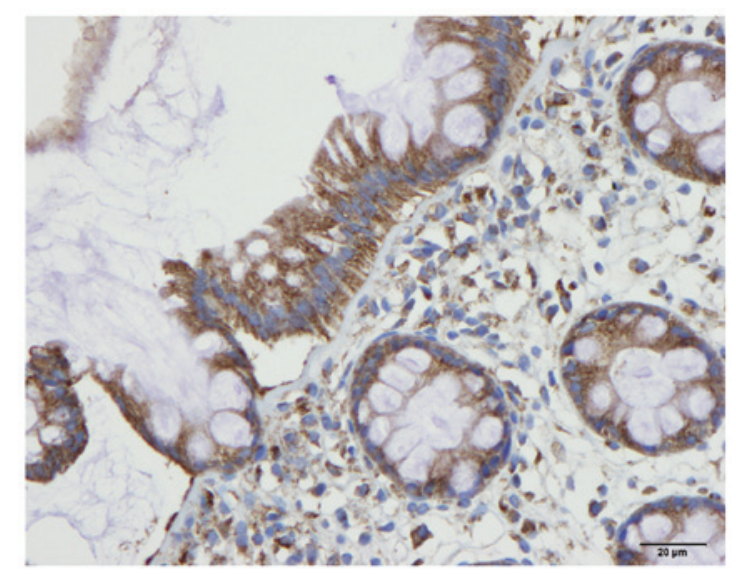

Figure 4. Serotonin-selective reuptake transporter immunoreactivity in the epithelial cells lining the rectal lumen in (A) a control subject and (B) in a patient with irritable bowel syndrome.

\section{Discussion}

Measurements of the immunoreactivity intensity of immunohistochemical staining as a function of the cellular content of certain substances has been attempted since computer image analysis started (44). The recent advances made in the computer software used in computer image analysis made it possible to obtain reliably measurements of the intensity of immunoreactivity reflecting the content of the cells of the immunostained substance. Intensity of immunoreactivity is expressed in arbitrary units and is useful only in comparisons between groups immunostained under the same conditions.

The present study showed that SERT immunoreactivity intensity decreased in the rectal epithelial cells of both IBS-D and IBS-C patients, indicating a reduction in SERT in these patients. This finding is in agreement with the previous observation (45) that SERT is decreased, as detected by both SERT messenger RNA and immunoreactivity intensity. However, the finding that rectal serotonin immunoreactivity intensity in IBS patients did not differ from that of the control, is not in agreement with earlier findings that serotonin content is decreased in the rectum of IBS patients (45). This discrepancy may be attributed to the difference in the methods used or to a difference in the patient cohort studied.

The main source of serotonin in the human body is the enterochromaffin cells in the gut mucosa (46-48). Serotonin is released by luminal acidification and pressure and acts on 
sensory and motor neurons in the submucosal and myenteric ganglia as well as in the spinal cord (46). This in turn stimulates motor, secretory and vasodilatory reflexes as well as afferent signals to the central nervous system (46). After exerting its effects at receptors, serotonin is transported by SERT into gut epithelial cells where it is degraded $(46,49)$. The gut mucosa has a high capacity for producing SERT as all the epithelial cells lining the luminal surface of the gut express SERT $(45,49)$. Reduction in SERT results in impaired intracellular uptake and degradation in the gut epithelial cells and consequently increases the availability of serotonin within the gut mucosa $(50,51)$. The previously published observations (45) and the present finding of reduced rectal SERT in the IBS patients could be one of the factors contributing to the development of both diarrhoea and constipation in these patients. In support of this assumption is that the use of selective serotonin reuptake inhibitors or exposure to desensitizing concentrations of exogenous serotonin can lead to either diarrhoea or constipation $(46,52)$. Moreover, transgenic mice lacking SERT exhibit alternating bowel habits between diarrhoea and constipation (53).

An increasing body of evidence suggests that a genetic abnormality concerning SERT occurs in IBS patients (54-63). The present study reported an abnormal expression of SERT in these patients and underlined the importance of SERT in the pathophysiology of IBS.

\section{Acknowledgements}

This study was supported by a grant from Helse-Fonna.

\section{References}

1. Thompson WG: A world view of IBS. In: Irritable Bowel Syndrome. Camilleri M and Spiller RC (eds.) Saunders, Philadelphia and London, pp17-26, 2002.

2. Drossman DA, Li Z, Andruzzi E, et al: U.S. householder survey of functional gastrointestinal disorders. Prevalence, sociodemography, and health impact. Dig Dis Sci 38: 1569-1580, 1993.

3. El-Salhy M, Gundersen D, Hatlebakk JG and Hausken T: Irritable Bowel Syndrome: Diagnosis, Pathogenesis and Treatment Options. Nova Science Publishers, Inc., New York, 2012.

4. Ford AC and Vandvik PO: Irritable bowel syndrome. Clin Evid (Online) 2012: pii0410 2012

5. Lovell RM and Ford AC: Global prevalence of and risk factors for irritable bowel syndrome: a meta-analysis. Clin Gastroenterol Hepatol 10: 712-721.e4, 2012.

6. Quigley EM, Locke GR, Mueller-Lissner S, et al: Prevalence and management of abdominal cramping and pain: a multinational survey. Aliment Pharmacol Ther 24: 411-419, 2006.

7. Vandvik PO, Lydersen S and Farup PG: Prevalence, comorbidity and impact of irritable bowel syndrome in Norway. Scand J Gastroenterol 41: 650-656, 2006.

8. Saito YA, Schoenfeld P and Locke GR 3rd: The epidemiology of irritable bowel syndrome in North America: a systematic review. Am J Gastroenterol 97: 1910-1915, 2002.

9. Whitehead WE, Burnett CK, Cook EW 3rd and Taub E: Impact of irritable bowel syndrome on quality of life. Dig Dis Sci 41: 2248-2253, 1996.

10. Agreus L, Svardsudd K, Nyren O and Tibblin G: Irritable bowel syndrome and dyspepsia in the general population: overlap and lack of stability over time. Gastroenterology 109: 671-680, 1995.

11. Thompson WG and Heaton KW: Functional bowel disorders in apparently healthy people. Gastroenterology 79: 283-288, 1980.

12. Kennedy TM, Jones RH, Hungin AP, O'Flanagan H and Kelly P: Irritable bowel syndrome, gastro-oesophageal reflux, and bronchial hyper-responsiveness in the general population. Gut 43: 770-774, 1998.
13. Talley NJ, Gabriel SE, Harmsen WS, Zinsmeister AR and Evans RW: Medical costs in community subjects with irritable bowel syndrome. Gastroenterology 109: 1736-1741, 1995.

14. Hungin AP, Whorwell PJ, Tack J and Mearin F: The prevalence, patterns and impact of irritable bowel syndrome: an international survey of 40,000 subjects. Aliment Pharmacol Ther 17: 643-650, 2003.

15. Jones R and Lydeard S: Irritable bowel syndrome in the general population. BMJ 304: 87-90, 1992.

16. Bordie AK: Functional disorders of the colon. J Indian Med Assoc 58: 451-456, 1972.

17. O'Keefe EA, Talley NJ, Zinsmeister AR and Jacobsen SJ: Bowel disorders impair functional status and quality of life in the elderly: a population-based study. J Gerontol A Biol Sci Med Sci 50: M184-M189, 1995.

18. Everhart JE and Renault PF: Irritable bowel syndrome in office-based practice in the United States. Gastroenterology 100: 998-1005, 1991.

19. Wilson S, Roberts L, Roalfe A, Bridge P and Singh S: Prevalence of irritable bowel syndrome: a community survey. Br J Gen Pract 54: 495-502, 2004.

20. Harvey RF, Salih SY and Read AE: Organic and functional disorders in 2000 gastroenterology outpatients. Lancet 1: 632-634, 1983.

21. Spiegel BM: The burden of IBS: looking at metrics. Curr Gastroenterol Rep 11: 265-269, 2009.

22. Longstreth GF, Thompson WG, Chey WD, Houghton LA, Mearin F and Spiller RC: Functional bowel disorders. Gastroenterology 130: 1480-1491, 2006.

23. Drossman DA: Rome III: the new criteria. Chin J Dig Dis 7: 181-185, 2006.

24. Harvey RF, Mauad EC and Brown AM: Prognosis in the irritable bowel syndrome: a 5-year prospective study. Lancet 1: 963-965, 1987.

25. Norgaard M, Farkas DK, Pedersen L, et al: Irritable bowel syndrome and risk of colorectal cancer: a Danish nationwide cohort study. Br J Cancer 104: 1202-1206, 2011.

26. Sloth $\mathrm{H}$ and Jorgensen LS: Chronic non-organic upper abdominal pain: diagnostic safety and prognosis of gastrointestinal and non-intestinal symptoms. A 5- to 7-year follow-up study. Scand J Gastroenterol 23: 1275-1280, 1988.

27. Pace F, Molteni P, Bollani S, et al: Inflammatory bowel disease versus irritable bowel syndrome: a hospital-based, case-control study of disease impact on quality of life. Scand J Gastroenterol 38: 1031-1038, 2003.

28. Gralnek IM, Hays RD, Kilbourne A, Naliboff B and Mayer EA: The impact of irritable bowel syndrome on health-related quality of life. Gastroenterology 119: 654-660, 2000.

29. Drossman DA, Morris CB, Schneck S, et al: International survey of patients with IBS: symptom features and their severity, health status, treatments, and risk taking to achieve clinical benefit. J Clin Gastroenterol 43: 541-550, 2009.

30. SandlerRS,Everhart JE, Donowitz M, et al: The burden of selected digestive diseases in the United States. Gastroenterology 122: 1500-1511, 2002.

31. Coates MD, Johnson AC, Greenwood-Van Meerveld B and Mawe GM: Effects of serotonin transporter inhibition on gastrointestinal motility and colonic sensitivity in the mouse. Neurogastroenterol Motil 18: 464-471, 2006.

32. Wang SH, Dong L, Luo JY, et al: Decreased expression of serotonin in the jejunum and increased numbers of mast cells in the terminal ileum in patients with irritable bowel syndrome. World J Gastroenterol 13: 6041-6047, 2007.

33. Kim HS, Lim JH, Park H and Lee SI: Increased immunoendocrine cells in intestinal mucosa of postinfectious irritable bowel syndrome patients 3 years after acute Shigella infection - an observation in a small case control study. Yonsei Med J 51: 45-51, 2010.

34. Lee KJ, Kim YB, Kim JH, Kwon HC, Kim DK and Cho SW: The alteration of enterochromaffin cell, mast cell, and lamina propria $\mathrm{T}$ lymphocyte numbers in irritable bowel syndrome and its relationship with psychological factors. J Gastroenterol Hepatol 23: 1689-1694, 2008.

35. Park JH, Rhee PL, Kim G, et al: Enteroendocrine cell counts correlate with visceral hypersensitivity in patients with diarrhoea-predominant irritable bowel syndrome. Neurogastroenterol Motil 18: 539-546, 2006.

36. Dunlop SP, Jenkins D, Neal KR and Spiller RC: Relative importance of enterochromaffin cell hyperplasia, anxiety, and depression in postinfectious IBS. Gastroenterology 125: $1651-1659,2003$ 
37. Spiller RC, Jenkins D, Thornley JP, et al: Increased rectal mucosal enteroendocrine cells, T lymphocytes, and increased gut permeability following acute Campylobacter enteritis and in post-dysenteric irritable bowel syndrome. Gut 47: 804-811, 2000.

38. El-Salhy M, Lomholt-Beck B and Hausken T: Chromogranin A as a possible tool in the diagnosis of irritable bowel syndrome. Scand J Gastroenterol 45: 1435-1439, 2010.

39. El-Salhy M, Mazzawi T, Gundersen D and Hausken T: Chromogranin A cell density in the rectum of patients with irritable bowel syndrome. Mol Med Rep 6: 1223-1225, 2012.

40. El-Salhy M, Lillebo E, Reinemo A and Salmelid L: Ghrelin in patients with irritable bowel syndrome. Int J Mol Med 23: 703-707, 2009.

41. Dizdar V, Spiller R, Singh G, et al: Relative importance of abnormalities of CCK and 5-HT (serotonin) in Giardia-induced post-infectious irritable bowel syndrome and functional dyspepsia. Aliment Pharmacol Ther 31: 883-891, 2010.

42. El-Salhy M, Vaali K, Dizdar V and Hausken T: Abnormal small-intestinal endocrine cells in patients with irritable bowel syndrome. Dig Dis Sci 55: 3508-3513, 2010.

43. El-Salhy M, Gundersen D, Ostgaard H, Lomholt-Beck B Hatlebakk JG and Hausken T: Low densities of serotonin and peptide YY cells in the colon of patients with irritable bowel syndrome. Dig Dis Sci 57: 873-878, 2012.

44. El-Salhy $M$ and Mahdavi J: Image analysis of the duodenal endocrine cells in mice with special reference to optic densitomertry. Ups J Med Sci 102: 175-184, 1997.

45. Coates MD, Mahoney CR, Linden DR, et al: Molecular defects in mucosal serotonin content and decreased serotonin reuptake transporter in ulcerative colitis and irritable bowel syndrome. Gastroenterology 126: 1657-1664, 2004.

46. Mawe GM, Coates MD and Moses PL: Review article: intestinal serotonin signalling in irritable bowel syndrome. Aliment Pharmacol Ther 23: 1067-1076, 2006.

47. Erspamer V: Occurrence and distribution of 5-hydroxytryptamine (enteramine) in the living organism. Z Vitam Horm Fermentforsch 9: 74-96, 1957.

48. Chen JX, Pan H, Rothman TP, Wade PR and Gershon MD: Guinea pig 5-HT transporter: cloning, expression, distribution, and function in intestinal sensory reception. Am J Physiol 275: G433-G448, 1998.

49. Spiller R and Lam C: An update on post-infectious irritable bowel syndrome: role of genetics, immune activation, serotonin and altered microbiome. J Neurogastroenterol Motil 18: 258-268, 2012.

50. Keating C, Beyak M, Foley S, et al: Afferent hypersensitivity in a mouse model of post-inflammatory gut dysfunction: role of altered serotonin metabolism. J Physiol 586: 4517-4530, 2008.
51. Coleman NS, Foley S, Dunlop SP, et al: Abnormalities of serotonin metabolism and their relation to symptoms in untreated celiac disease. Clin Gastroenterol Hepatol 4: 874-881, 2006.

52. Wade PR, Chen J, Jaffe B, Kassem IS, Blakely RD and Gershon MD: Localization and function of a 5-HT transporter in crypt epithelia of the gastrointestinal tract. J Neurosc 16: 2352-2364, 1996

53. Chen JJ, Li Z, Pan H, et al: Maintenance of serotonin in the intestinal mucosa and ganglia of mice that lack the high-affinity serotonin transporter: abnormal intestinal motility and the expression of cation transporters. J Neurosci 21: 6348-6361, 2001.

54. Faure C, Patey N, Gauthier C, Brooks EM and Mawe GM: Serotonin signaling is altered in irritable bowel syndrome with diarrhea but not in functional dyspepsia in pediatric age patients. Gastroenterology 139: 249-258, 2010.

55. Kim HJ, Camilleri M, Carlson PJ, et al: Association of distinct alpha(2) adrenoceptor and serotonin transporter polymorphisms with constipation and somatic symptoms in functional gastrointestinal disorders. Gut 53: 829-837, 2004.

56. Camilleri M, Andrews CN, Bharucha AE, et al: Alterations in expression of p11 and SERT in mucosal biopsy specimens of patients with irritable bowel syndrome. Gastroenterology 132: $17-25,2007$.

57. Camilleri M, Busciglio I, Carlson P, et al: Candidate genes and sensory functions in health and irritable bowel syndrome. Am J Physiol Gastrointest Liver Physiol 295: G219-G225, 2008.

58. Kumar S, Ranjan P, Mittal B and Ghoshal UC: Serotonin transporter gene (SLC6A4) polymorphism in patients with irritable bowel syndrome and healthy controls. J Gastrointestin Liver Dis 21: 31-38, 2012.

59. Park JM, Choi MG, Park JA, et al: Serotonin transporter gene polymorphism and irritable bowel syndrome. Neurogastroenterol Motil 18: 995-1000, 2006

60. Saito YA, Locke GR 3rd, Zimmerman JM, et al: A genetic association study of 5-HTT LPR and GNbeta3 C825T polymorphisms with irritable bowel syndrome. Neurogastroenterol Motil 19: 465-470, 2007

61. Colucci R, Blandizzi C, Bellini M, Ghisu N, Tonini M and Del Tacca M: The genetics of the serotonin transporter and irritable bowel syndrome. Trends in molecular medicine 14: 295-304, 2008

62. Colucci R, Gambaccini D, Ghisu N, et al: Influence of the serotonin transporter 5HTTLPR polymorphism on symptom severity in irritable bowel syndrome. PloS One 8: e54831, 2013.

63. Park MI and Camilleri M: Is there a role of food allergy in irritable bowel syndrome and functional dyspepsia? A systematic review. Neurogastroenterol Motil 18: 595-607, 2006. 